more common in older patients, those with a past history of FS, and patients with a significantly lower body temperature at seizure occurrence $(39.29+/-0.61 \mathrm{C}$ vs $39.57+/-0.61 \mathrm{C}$, $\mathrm{P}=0.032$ ). PPIC was associated with a higher body temperature at seizure occurrence $(39.80+/-0.65 \mathrm{C}$ vs $39.46+/-0.60 \mathrm{C}, \mathrm{P}=0.011)$, intravenous administration of diazepam $(9 / 28$ [32.1\%] vs $4 / 187$ [2.1\%], $\mathrm{P}<0.0001)$, and prolonged seizure $(10 / 28$ [35.7\%] vs $5 / 187[2.7 \%]$, $\mathrm{P}<0.0001$ ). (Hara K, Tanabe T, Aomatsu T, et al. Febrile seizures associated with influenza A. Brain Dev Jan 2007;29:30-38). (Respond: Dr Keita Hara, Division of Pediatrics, Hirakata City Hospital, Osaka, Japan; e-mail: harakatacity@tree.odn.ne.jp).

COMMENT. An almost identical study and similar findings to those in Japan are reported from Hong Kong (Kwong KL et al. Pediatr Neurol Dec 2006;35:395-399; see Ped Neur Briefs Dec 2006;20:94-5).

In the Japanese study, FS were complex in one third of the influenza-associated seizures, and postictal impairment of consciousness was prolonged (PPIC) for more than 30 $\min$ in $13 \%$. Children with FS generally recover consciousness in less than $30 \mathrm{~min}$. If recovery of consciousness takes $>1$ hour, an acute symptomatic etiology should be suspected, according to a recent UK report (Allen JE et al. Arch Dis Child Jan 2007;92:3942; Ped Neur Briefs Jan 2007;21:4). In the simultaneous Japanese report, none of the influenza FS patients had evidence of encephalitis or structural brain lesion. However, many had complex FS, and PPIC was associated with prolonged seizure and with the need for administration of diazepam. Those with partial FS had a lower body temperature, indicative of a lower FS threshold (Millichap JG. Pediatrics 1959;23:76-85). Patients with a higher body temperature had PPIC and had received diazepam, given to raise the seizure threshold. A significant factor for development of FS in influenza is a coexisting gastroenteritis (Kwong KL et al. Ped Neur Briefs Dec 2006;20:94-5), a cause of afebrile seizures with focal onset, as described in the following article.

\title{
MILD VIRAL GASTROENTERITIS AND AFEBRILE SEIZURES
}

Ictal electroencephalograms (EEGs) were recorded in six patients ( 2 male, 4 female; ages 14 mo to $38 \mathrm{mo}$ ) with afebrile convulsions and mild gastroenteritis (CwG), in a study at Nagoya, Japan. None had febrile convulsions. A family history of $\mathrm{CwG}$ was positive in 2 patients, and the twin of one patient had a history of febrile seizures. A cluster of seizures occurred within $1-3$ days of the onset of gastroenteritis, Rotavirus antigen test in the stool was positive in 2 patients. Seizures began as partial, and clinical manifestations included loss of responsiveness, arrest of movement, cyanosis, lateral eye deviation, and hemifacial convulsion. A generalized tonic-clonic convulsion occurred in 5 patients. Ictal EEGs showed focal low amplitude fast activity, beginning in the occipital area in 3 patients, and parietal, central, or frontal in the remaining 3. The focal discharge evolved into a secondary generalized seizure. Seizures were controlled with phenobarbital, lidocaine, or carbamazepine. At follow-up (mean $39 \mathrm{mo}$, range 17-62 mo) interictal EEGs and psychomotor development were normal. (Maruyama K, Okumura A, Sofue A, Ishihara N, Watanabe K. Ictal EEG in patients with convulsions with mild gastroenteritis. Brain Dev Jan 2007;29:43-46). (Respond: Dr Koichi Maruyama, Department of Pediatrics, Anjo Kosei Hospital, Aichi 446-8602, Japan). 
COMMENT. Convulsion with mild gastroenteritis $(\mathrm{CwG})$ is a frequent seizure disorder in Japan (Uemura $N$ et al. Brain Dev 2002;24:745-9). Previously described as generalized, the seizures appear to be partial with secondary generalization. CwG presents in previously healthy infants or young children aged 6 months to 3 years. Seizures are afebrile and occur in clusters in close relation to the onset of diarrhea. Laboratory results including electrolytes, glucose, and CSF are normal, ictal EEG is focal and secondary generalized, the interictal EEG is normal, and the outcome benign. In the above study, no reports of neuroimaging are included. Rotavirus was the likely cause in 2 of the 6 patients.

Usually affecting Japanese children, CwG is now known to occur outside Asia. A study of 14 children with the syndrome is reported from the UK (Narchi H. Benign afebrile cluster convulsions with gastroenteritis. BMC Pediatr 2004 Feb 5;4:2 [abstract]). The gastroenteritis was considered viral, and Rotavirus was detected by enzyme immunoassay in the stools of 7 of 10 patients tested. Stool culture was negative for pathogenic bacteria. CSF and computed tomography, performed on 2 patients with prolonged seizures, were negative. On long-term follow-up, none had recurrence of seizures and all had normal development. The seizure mechanism is unknown, and CNS complications are not generally described with Rotavirus infection (AAP Redbook). In the absence of fever, electrolyte and glucose abnormalities, a viral neurotropism, similar to that suggested for HHV-6 in febrile seizures (Hall CB et al, 1994; Millichap JG and JJ, 2006), might be considered.

\section{INFECTIOUS MONONUCLEOSIS AND MULTIPLE SCLEROSIS}

A cohort of 25,234 Danish patients testing positive (at titers of 1/32) for Epstein-Barr virus related mononucleosis (MONO), during the period 1940-1988, was followed for more than 550,000 person-years. The cohort was linked to the Multiple Sclerosis (MS) Registry for determination of risk of developing MS, in a study at Statens Serum Institut, Copenhagen. A total of 104 cases of MS observed vs 46 expected in the cohort testing positive for MONO corresponded to a standardized incidence ratio (SIR) of 2.27. In a cohort with negative MONO test, 68 observed cases corresponded to a SIR of 1.23, and similar to that prevailing in the general population. Elevated SIRs for MS were observed for MONO patients at all ages, except those 30 years or older. SIR for MS diagnosis was inversely correlated with age at MONO infection ( $\mathrm{P}$ for trend $=0.03$ ). Increased risk of MS appeared 10 years or more after MONO occurred and remained increased for more than 3 decades. The risk was uniformly distributed for sex and age; it was not correlated with severity of MONO infection. (Nielsen TR, Rostgaard K, Nielsen NM, et al. Multiple sclerosis after infectious mononucleosis. Arch Neurol Jan 2006;64:72-75). (Respond: TR Nielsen MD, Department of Epidemiology Research, Statens Serum Institut, Artillerivej 5, DK-2300 Copenhagen S, Denmark).

COMMENT. After recovery from infectious mononucleosis, a patient has a greater than 2-fold increased risk of developing multiple sclerosis. The risk of MS is increased soon after mononucleosis and extends for up to 30 years; it is independent of sex, age, and time from infection or severity of infection.

The association of 3-methylcrotonyl-CoA carboxylase deficiency and severe multiple sclerosis is reported in a 13-year-old female (Darin $\mathrm{N}$ et al. Pediatr Neurol Feb 2007;36:132-134). She presented with a mild Reye-like syndrome following influenza A 